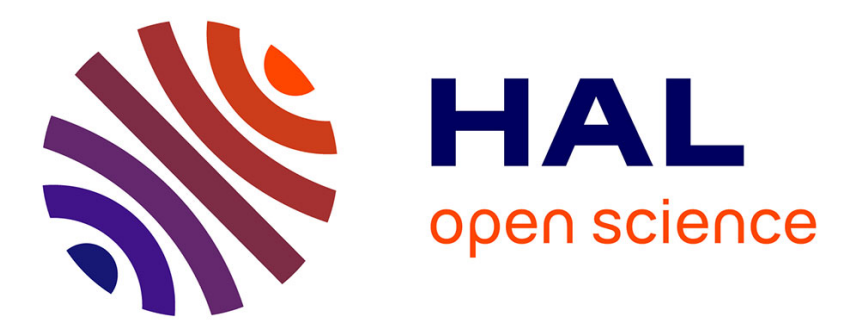

\title{
Synthesis of 1,4-disubstituted 1,2,3-triazole Derivatives Using Click Chemistry and their Src Kinase Activities
}

Alexandre Lebeau, Cyril Abrioux, David Bénimèlis, Zohra Benfodda, Patrick Meffre

\section{- To cite this version:}

Alexandre Lebeau, Cyril Abrioux, David Bénimèlis, Zohra Benfodda, Patrick Meffre. Synthesis of 1,4disubstituted 1,2,3-triazole Derivatives Using Click Chemistry and their Src Kinase Activities. Medicinal Chemistry, 2017, Medicinal Chemistry, 13, pp.40-48. 10.2174/1573406412666160404125718. hal-01495029

\author{
HAL Id: hal-01495029 \\ https://hal.science/hal-01495029
}

Submitted on 12 Jul 2017

HAL is a multi-disciplinary open access archive for the deposit and dissemination of scientific research documents, whether they are published or not. The documents may come from teaching and research institutions in France or abroad, or from public or private research centers.
L'archive ouverte pluridisciplinaire HAL, est destinée au dépôt et à la diffusion de documents scientifiques de niveau recherche, publiés ou non, émanant des établissements d'enseignement et de recherche français ou étrangers, des laboratoires publics ou privés.

\section{(ㅇ)(1) $\$$}

Distributed under a Creative Commons Attribution - NonCommercial - NoDerivatives| 4.0 


\title{
Synthesis of 1,4-disubstituted 1,2,3-triazole Derivatives Using Click Chemistry and their Src Kinase Activities
}

\author{
Alexandre Lebeau, Cyril Abrioux, David Bénimèlis, Zohra Benfodda ${ }^{\# *}$ and Patrick Meffre ${ }^{\#}$
}

UNIV. NIMES, EA7352 CHROME, Rue du Dr G. Salan, 30021 Nîmes cedex 1, France

\begin{abstract}
A series of 1,4-disubstituted 1,2,3-triazoles derivatives (3) were designed and prepared as potential inhibitors for Src kinase. In this manuscript, all of the designed compounds were screened via molecular docking using PLANTS as virtual screening software to identify new inhibitors of Src kinase. As expected, the results of the docking study revealed that most of these targeted compounds possessed low binding energy. Subsequently, all of the screened compounds were synthesized via Huisgen's 1,3-dipolar cycloaddition between terminal alkynes (1) and methyl 2-azidoacetate (2) with $\mathrm{Cu}(\mathrm{I})$ in excellent yields at room temperature. The synthesized triazoles (3) were characterized by $\mathrm{IR},{ }^{1} \mathrm{H},{ }^{13} \mathrm{C},{ }^{19} \mathrm{~F}$ NMR and mass spectral techniques and they were investigated as inhibitors of Src kinase.

Among these compounds, $\mathbf{3 m}$ exhibited the most potent inhibitory activity against Src kinase.
\end{abstract}

Keywords: 1,2,3-triazole, molecular docking, Huisgen's 1,3-dipolar cycloaddition, PEG400, Click chemistry, Src kinase.

\section{INTRODUCTION}

Tyrosine kinases (TK) are enzymes that catalyze the phosphorylation of Tyrosine residues on proteins by the transfer of phosphate moiety of ATP. TK are key regulators of various cell functions, such as cellular growth, proliferation, migration, differentiation, and apoptosis [1]. The Src family of kinases (SFKs) are non-receptor tyrosine kinases. Since Src is involved in several cell signaling pathways, inhibition of Src activity could be effective for the treatment of various diseases, including cancer [2,3]. Src mutations and/or overexpression has been correlated with tumor growth, metastasis, and angiogenesis $[4,5]$. Thus, the design and the discovery of novel Src kinase inhibitors remains critically important.

Quinoline carbonitriles [6-9], ATP-phosphopeptide conjugates [10], pyrazolopyrimidines [11], purines [12,13], imidazo[1,5-a]-pyrazines [14], benzotriazines [15], pyrimidoquinolines [16], pyridopyrimidinones [17], quinazolines [18] and more recently 1,4-disubstituted 1,2,3-triazoles [19] have been reported to target Src Kinase.

There has been a great interest in the development of five-membered nitrogen heterocycles in medicinal chemistry. Among them, 1,2,3-triazole heterocycles containing molecules are reported to possess a large biological spectrum as antimicrobial [2022], antimalarial [23], antihistaminic

[24], antifungal [25,26], anti-Hiv [27,28], antitubercular [29]

\footnotetext{
*Address correspondence to this author at Nîmes University, EA 7352 CHROME, 30021, Nîmes, France; Tel: +33466279589, Fax:

+33466364587; E-mail: zohra.benfodda@unimes.fr

"These authors contributed equally to this work
}

and anticancer agents [30,31]. Good stability and high aqueous solubility of these compounds in biological systems are appreciable. Moreover, this scaffold can be tuned to form powerful pharmacophores and also play an important role in bioconjugation [32].

Huisgen's cycloaddition, the general method for the synthesis of 1,4-disubstituted 1,2,3-triazoles, involves a 1,3dipolar cycloaddition between azides and alkynes under thermal conditions to afford an equal mixture of 1,4- and 1,5-disubstituted isomers [33]. Sharpless and co-workers have reported remarkable high-yielding syntheses with excellent regioselectivity [34,35]. The $\mathrm{Cu}(\mathrm{I})$ catalyzed Huisgen 1,3-dipolar cycloaddition of azides and terminal alkynes is the model example of a click chemistry, to give only 1,4disubstituted 1,2,3-triazoles. 
In the present study, we report the design and the synthesis of a series of 1,4-disubstituted 1,2,3-triazoles involving one pot condensation of methyl 2-azidoacetate and different terminal alkynes. All the synthesized triazoles were characterized by IR, ${ }^{1} \mathrm{H},{ }^{13} \mathrm{C},{ }^{19} \mathrm{~F}$ NMR, and HRMS. They were investigated as inhibitors of Src kinase.

\section{EXPERIMENTAL}

\subsection{Chemistry}

All reagents were purchased from the Aldrich Chemical Co. and used without any purification. Solvents were distilled from the appropriate drying agents immediately prior to use. ${ }^{1} \mathrm{H},{ }^{19} \mathrm{~F}$, and ${ }^{13} \mathrm{C}$ NMR spectra were recorded at $300.13 \mathrm{MHz}, 282.37$ $\mathrm{MHz}$ and $75.46 \mathrm{MHz}$ respectively with a Brucker Avance 300 spectrometer; the chemical shifts are given in ppm relative to $\mathrm{Me}_{4} \mathrm{Si}$ for the ${ }^{1} \mathrm{H}$ and ${ }^{13} \mathrm{C}$, and $\mathrm{CCl}_{3} \mathrm{~F}$ for ${ }^{19} \mathrm{~F}$, as internal standards. Data are reported as follows: chemical shifts, multiplicity (s $=$ singlet, $\mathrm{d}=$ doublet, $\mathrm{t}=$ triplet, $\mathrm{q}=$ quartet, $\mathrm{br}=$ broad, $\mathrm{dd}=$ double doublet, $\mathrm{dt}=$ double of triplet, $\mathrm{m}=$ multiplet $)$, coupling constants (Hz), integration. High resolution mass spectra (HRMS) were obtained by electrospray using a TOF analyzer Platform. IR spectra were obtained using a Jasco FT-IR 410 instrument $(\mathrm{cm} 1)$ as a thin film on $\mathrm{NaCl}$ disc as stated. Reactions

were monitored with Merck Kieselgel $60 \mathrm{~F}_{254}$ precoated aluminium silica gel plates $(0.25 \mathrm{~mm}$ thickness). Melting points were determined on a Stuart scientific SMP10 apparatus and are uncorrected. Flash chromatographies were performed on a Grace Reveleris X2 using 40m packed silica cartridge. Purity of the compounds were determinate by HPLC-UV at $254 \mathrm{~nm}$ Flash chromatographies experiments were carried out on Silica Gel premium Rf grade (40-63 m) or were performed on a Grace Reveleris X2 using 40m packed silica cartridge. HPLC analyses were obtained on the Waters Alliance 2795 using the following conditions : thermo Hypersil C18 column ( $3 \mathrm{~m}, 50 \mathrm{~mm} \mathrm{~L}$ x $2.1 \mathrm{~mm} \mathrm{ID}), 20^{\circ} \mathrm{C}$ column temperature, $0.2 \mathrm{~mL} / \mathrm{min}$ flow rate, photodiodearray detection $(210400 \mathrm{~nm}$ ), mobile phase consistent of a gradient of water and acetonitrile (each containing $0.1 \%$ trifluoroacetic acid).

\subsection{General Synthetic Procedure : Huisgen's 1,3-Dipolar Cycloaddition of Azides and Alkynes}

Method A: Alkyne (1.00 eq.) and azide (1.00 eq.) were dissolved in $\mathrm{tBuOH} / \mathrm{H}_{2} \mathrm{O}(1: 1, \mathrm{v} / \mathrm{v})$. Solid $\mathrm{CuI}$ was added $(0.05$ eq., $5 \mathrm{~mol} \%)$ and solid sodium ascorbate $(0.10$ eq., $10 \mathrm{~mol} \%)$ were added. The reaction mixture was stirred at room temperature for 12 to $72 \mathrm{~h}$. Reactions were monitored by TLC and when the reaction is finished ethyl acetate was added. The mixture was extracted three times with ethyl acetate, the organic phases were washed with saturated $\mathrm{NaCl}$, combined and dried over $\mathrm{MgSO}_{4}$. EtOAc was evaporated under reduced pressure to give the crude product. The residue was purified by flash column chromatography (silica gel, EtOAc:Cyclohexane $=1: 9$ ramping to EtOAc) to give the desired triazoles.

Method B: Alkyne (1.00 eq.) and azide (1.00 eq.) were dissolved PEG 400/ $\mathrm{H}_{2} \mathrm{O}(1: 1, \mathrm{v} / \mathrm{v}, 2 \mathrm{~mL})$. Solid $\mathrm{CuSO}_{4}$ was added $(0.05$ eq., $5 \mathrm{~mol} \%)$ and solid sodium ascorbate $(0.10$ eq., $10 \mathrm{~mol} \%)$ were added. The reaction mixture was allowed to stir at room temperature for $1-8 \mathrm{~h}$. After the reaction was complete, as indicated by TLC, the reaction mixture was diluted with water and filtered at the pump to collect the product or extracted with ethyl acetate $(32 \mathrm{ml})$. The organic layer was dried over anhydrous sodium sulfate and distilled using rotary vacuum evaporator. The afforded crude product was recrystallized from cyclohexane.

\subsubsection{Synthesis of methyl 2-(4-(2-aminophenyl)-1H-1,2,3triazol-1-yl)acetate (3a)}

$\mathrm{R}_{f}=0.4\left(\right.$ Cyclohexane/EtOAc, 1:1, v/v); Yellow solid; mp: 113-115 ${ }^{\circ} \mathrm{C}$; IR: $3452(\mathrm{NH}), 3333(\mathrm{NH}), 1753(\mathrm{C}=\mathrm{O}$ ester). Yield=99\%; ${ }^{1} \mathrm{H}$ NMR (300.13 MHz, DMSO) $8.51(\mathrm{~s}, 1 \mathrm{H}, \mathrm{C}=\mathrm{CH}-\mathrm{N}), 7.44(\mathrm{dd}, \mathrm{J}=7.7,1.1 \mathrm{~Hz}, 1 \mathrm{H}, \mathbf{H a r}), 7.16-6.92(\mathrm{~m}, 1 \mathrm{H}$, Har), $6.78(\mathrm{~d}, \mathrm{~J}=8.0 \mathrm{~Hz}, 1 \mathrm{H}$, Har), $6.60(\mathrm{t}, \mathrm{J}=$

$7.4 \mathrm{~Hz}, 1 \mathrm{H}, \mathbf{H a r}), 6.17$ (s, 2H, C-NH2), 5.48 (s, 2H, N-CH2 $\mathrm{C}=\mathrm{O}), 3.74$ (s, 3H, O-CH3); ${ }^{13} \mathrm{C}$ NMR (75 MHz, DMSO) 167.6 $(\mathbf{C}=\mathrm{O}), 147.3(\mathrm{C}-\mathbf{C}-\mathrm{N}=), 145.6(\mathbf{C a r}), 128.5(\mathbf{C H a r}), 127.5(\mathbf{C H a r}), 122.6(\mathrm{C}=\mathbf{C H}-\mathrm{N}), 116(\mathbf{C H a r}), 115.8(\mathbf{C H a r}), 112.4(\mathbf{C a r})$, $52.6\left(\mathrm{O}-\mathrm{CH}_{3}\right), 50.4\left(\mathrm{~N}-\mathrm{CH}_{2}\right)$. HRMS $\left(\mathrm{M}+\mathrm{H}^{+}\right): 233.1039$ calcd for $\mathrm{C}_{11} \mathrm{H}_{12} \mathrm{~N}_{4} \mathrm{O}_{2} ; 233.1038$ found.

\subsubsection{Synthesis of methyl 2-(4-(3-aminophenyl)-1H-1,2,3triazol-1-yl)acetate (3b)}

$\mathrm{R}_{f}=0.1\left(\right.$ Cyclohexane/EtOAc, 1:1, v/v); Pale brown solid; mp: 98-100 ${ }^{\circ} \mathrm{C}$; IR: $3431(\mathrm{NH}), 3350(\mathrm{NH}), 3135(\mathrm{CHar}) 1737$ $\left(\mathrm{C}=\mathrm{O}\right.$ ester); Yield $=100 \%$; ${ }^{1} \mathrm{H}$ NMR $(300.13 \mathrm{MHz}, \mathrm{DMSO}) 8.39(\mathrm{~s}, 1 \mathrm{H}, \mathrm{C}=\mathrm{CH}-\mathrm{N}), 7.12-7.04(\mathrm{~m}, \mathrm{~J}=10.2,5.0 \mathrm{~Hz}, 2 \mathrm{H}$, Har), $6.93(\mathrm{~d}, \mathrm{~J}=7.6 \mathrm{~Hz}, 1 \mathrm{H}$,

Har), 6.53 (dd, J = 7.9, $1.3 \mathrm{~Hz}, 1 \mathrm{H}, \mathbf{H a r}), 5.44$ (s, 2H, NCH $2-\mathrm{C}=\mathrm{O}), 5.21$ (s, 2H, C-NH2), 3.73 (s, 3H, O-CH3); ${ }^{13} \mathrm{C}$ NMR (75 MHz, DMSO) 167.7 (C=O), 149.1 (C-C-N=), 147 (Car), 131.1 (Car), $129.4(\mathbf{C H a r}), 122.2(\mathrm{C}=\mathbf{C H}-\mathrm{N})$, 113.6 (CHar), 113.2 ( CHar), $110.4(\mathbf{C H a r}), 52.5\left(\mathrm{O}_{-} \mathrm{CH}_{3}\right), 50.3\left(\mathrm{~N}-\mathrm{CH}_{2}\right)$. HRMS $\left(\mathrm{M}+\mathrm{H}^{+}\right)$: 233.1039 calcd for $\mathrm{C}_{11} \mathrm{H}_{12} \mathrm{~N}_{4} \mathrm{O}_{2} ; 233.1036$ found.

\subsubsection{Synthesis of methyl 2-(4-(4-aminophenyl)-1H-1,2,3triazol-1-yl)acetate (3c)}


$\mathrm{R}_{f}=0.1\left(\right.$ Cyclohexane/EtOAc, 1:1, v/v); Yellow solid; mp: 147-149 ${ }^{\circ} \mathrm{C}$; IR: $3429(\mathrm{NH}), 3357(\mathrm{NH}), 3108$ (CHar) 1745 $\left(\mathrm{C}=\mathrm{O}\right.$ ester); Yield $=70 \% ;{ }^{1} \mathrm{H}$ NMR $(300.13 \mathrm{MHz}, \mathrm{DMSO}) 8.25(\mathrm{~s}, 1 \mathrm{H}, \mathrm{C}=\mathrm{CH}-\mathrm{N}), 7.49(\mathrm{~d}, \mathrm{~J}=8.4 \mathrm{~Hz}$,

$2 \mathrm{H}, \mathbf{H a r}), 6.61$ (d, J = 8.4 Hz, 2H, Har), 5.40 (s, 2H, NCH $2-\mathrm{C}=\mathrm{O}), 5.26\left(\mathrm{~s}, 2 \mathrm{H}, \mathrm{C}-\mathrm{NH}_{2}\right), 3.72(\mathrm{~s}, 3 \mathrm{H}, \mathrm{O}-\mathrm{CH}) ;{ }^{13} \mathrm{C}$

NMR (75 MHz, DMSO) 167.8 (C=O), 148.7 (C-C-N=), 147.3 (Car), 126.1 (CHar), 120.5 (C=CH-N), 118 (Car), 113.9 (CHar), $52.5\left(\mathrm{O}-\mathrm{CH}_{3}\right), 50.3\left(\mathrm{~N}-\mathrm{CH}_{2}\right)$. HRMS $\left(\mathrm{M}+\mathrm{H}^{+}\right): 233.1039$ calcd for $\mathrm{C}_{11} \mathrm{H}_{12} \mathrm{~N}_{4} \mathrm{O}_{2} ; 233.1040$ found.

\subsubsection{Synthesis of methyl 2-(4-(o-tolyl)-1H-1,2,3-triazol-1yl)acetate (3d)}

$\mathrm{R}_{f}=0.5$ (Cyclohexane/EtOAc, 1:1, v/v); White solid; mp: 66-68 ${ }^{\circ} \mathrm{C}$; IR: 3146 ( CHar), 1755 (C=O ester); Yield = 27\%; ${ }^{1} \mathrm{H}$ NMR (300.13 MHz, DMSO) $8.42(\mathrm{~s}, 1 \mathrm{H}, \mathrm{C}=\mathrm{CHN}), 7.86-7.65(\mathrm{~m}, 1 \mathrm{H}, \mathbf{H a r}), 7.44-7.15(\mathrm{~m}, 3 \mathrm{H}, \mathbf{H a r}), 5.48(\mathrm{~s}, 2 \mathrm{H}$, $\mathrm{N}-\mathrm{CH}_{2}-\mathrm{C}=\mathrm{O}$ ), 3.74 (s, 3H, O-CH 3 ), 2.43 (s, 3H, C-CH3); ${ }^{13} \mathrm{C}$ NMR (75 MHz, DMSO) 167.7 (C=O), 145.5 (C-C-N=), 134.8 (Car), 130.9 (CHar), 129.7 (Car), 128 (CHar), 127.8 (CHar), 126.1 (CHar), 124.7 (C=CH-N), 52.5

$\left(\mathrm{O}-\mathrm{CH}_{3}\right), 50.3\left(\mathrm{~N}-\mathrm{CH}_{2}\right), 21.1\left(\mathrm{Car}-\mathrm{CH}_{3}\right)$. HRMS $\left(\mathrm{M}+\mathrm{H}^{+}\right)$: 232.1086 calcd for $\mathrm{C}_{12} \mathrm{H}_{14} \mathrm{~N}_{3} \mathrm{O}_{2} ; 232.1089$ found.

\subsubsection{Synthesis of methyl 2-(4-(m-tolyl)-1H-1,2,3-triazol-1yl)acetate (3e)}

$\mathrm{R}_{f}=0.5$ (Cyclohexane/EtOAc, 1:1, v/v); White solid; mp: 64-66 ${ }^{\circ} \mathrm{C}$; IR: 3127 ( CHar), 1747 (C=O ester); Yield = 60\%; ${ }^{1} \mathrm{H}$ NMR (300.13 MHz, DMSO) 8.54 (s, 1H, C=CHN), 7.70 (s, 1H, Har), 7.64 (d, J = 7.7 Hz, 1H, Har), 7.34 (t, J = 7.6 $\mathrm{Hz}, 1 \mathrm{H}, \mathrm{Har}), 7.16(\mathrm{~d}, \mathrm{~J}=7.5 \mathrm{~Hz}, 1 \mathrm{H}, \mathrm{Har}), 5.47(\mathrm{~s}$,

$\left.2 \mathrm{H}, \mathrm{N}-\mathrm{CH}_{2}-\mathrm{C}=\mathrm{O}\right), 3.74\left(\mathrm{~s}, 3 \mathrm{H}, \mathrm{O}-\mathrm{CH}_{3}\right), 2.36\left(\mathrm{~s}, 3 \mathrm{H}, \mathrm{CCH}_{3}\right) ;{ }^{13} \mathrm{C}$ NMR (75 MHz, DMSO) $167.7(\mathbf{C}=\mathrm{O}), 146.4(\mathrm{CC}-\mathrm{N}=), 138.1$ (Car), $130.4(\mathbf{C a r}), 128.8$ (CHar), $128.6(\mathbf{C H a r}), 125.7(\mathbf{C H a r}), 122.6(\mathrm{C}=\mathbf{C H}-\mathrm{N}), 122.3(\mathbf{C H a r}), 52.6\left(\mathrm{O}-\mathbf{C H}_{3}\right), 50.4\left(\mathrm{~N}_{-} \mathbf{C H}_{2}\right)$, $21\left(\mathrm{Car}-\mathrm{CH}_{3}\right)$. HRMS $\left(\mathrm{M}+\mathrm{H}^{+}\right)$:

232.1086 calcd for $\mathrm{C}_{12} \mathrm{H}_{14} \mathrm{~N}_{3} \mathrm{O}_{2} ; 232.1087$ found.

\subsubsection{Synthesis of methyl 2-(4-(p-tolyl)-1H-1,2,3-triazol-1yl)acetate (3f)}

$\mathrm{R}_{f}=0.5$ (Cyclohexane/EtOAc, 1:1, v/v); White solid; mp 118-120 ${ }^{\circ} \mathrm{C}$, lit: 116-117 ${ }^{\circ} \mathrm{C}$ [36]; IR: 3135 ( CHar), $1760(\mathrm{C}=\mathrm{O}$ ester); Yield = 54\%; ${ }^{1} \mathrm{H}$ NMR (300.13 MHz, DMSO) $8.51(\mathrm{~s}, 1 \mathrm{H}, \mathrm{C}=\mathrm{CH}-\mathrm{N}), 7.75(\mathrm{~d}, \mathrm{~J}=8.0 \mathrm{~Hz}, 2 \mathrm{H}, \mathbf{H a r}), 7.27$ (d, J = 8.0 $\mathrm{Hz}, 2 \mathrm{H}, \mathbf{H a r}), 5.46$ (s, 2H, N-CH2 $-\mathrm{C}=\mathrm{O}$ ), 3.74 (s, 3H, O-CH3), 2.33 (s, 3H, C-CH3); ${ }^{13} \mathrm{C}$ NMR (75 MHz, DMSO) 167.7 $(\mathbf{C}=\mathrm{O}), 146.4(\mathrm{C}-\mathbf{C}-\mathrm{N}=), 137.3(\mathbf{C a r}), 129.5(\mathbf{C H a r}), 127.7(\mathbf{C a r}), 125.1(\mathbf{C H a r}), 122.3(\mathrm{C}=\mathbf{C H}-\mathrm{N}), 52.5$

$\left(\mathrm{O}-\mathrm{CH}_{3}\right), 50.4\left(\mathrm{~N}-\mathrm{CH}_{2}\right), 20.8\left(\mathrm{Car}-\mathrm{CH}_{3}\right)$. HRMS $\left(\mathrm{M}+\mathrm{H}^{+}\right)$: 232.1086 calcd for $\mathrm{C}_{12} \mathrm{H}_{14} \mathrm{~N}_{3} \mathrm{O}_{2} ; 232.1087$ found.

\subsubsection{Synthesis of methyl 2-(4-(2-chlorophenyl)-1H-1,2,3triazol-1-yl)acetate (3g)}

$\mathrm{R}_{f}=0.6$ (Cyclohexane/EtOAc, 1:1, v/v); White solid; mp 69-71 ${ }^{\circ} \mathrm{C}$; IR: $3145(\mathrm{CHar}), 1741(\mathrm{C}=\mathrm{O}$ ester); Yield = 49\%; ${ }^{1} \mathrm{H}$ NMR (300.13 MHz, DMSO) $8.73(\mathrm{~s}, 1 \mathrm{H}, \mathrm{C}=\mathrm{CHN}), 8.13$ (dd, J = 7.6, 1.9 Hz, 1H, Har), 7.59 (dd, J = 7.8, 1.2 $\mathrm{Hz}, 1 \mathrm{H}, \mathbf{H a r}), 7.52-7.36$ (m, 2H, Har), 5.51 (s, 2H, NCH2 $-\mathrm{C}=\mathrm{O}$ ), 3.74 (s, 3H, O-CH3); ${ }^{13} \mathrm{C}$ NMR (75 MHz, DMSO) 167.7 $(\mathbf{C}=\mathrm{O}), 142.5(\mathrm{C}-\mathbf{C}-\mathrm{N}=), 130.3$ (CHar), 130.2 (Car), $129.5(\mathbf{C H a r}), 129.4(\mathbf{C H a r}), 128.9(\mathbf{C a r}), 127.6(\mathbf{C H a r}), 125.7(\mathrm{C}=\mathbf{C H}-$ $\mathrm{N}), 52.6\left(\mathrm{O}-\mathrm{CH}_{3}\right), 50.4\left(\mathrm{~N}-\mathrm{CH}_{2}\right)$. HRMS $\left(\mathrm{M}+\mathrm{H}^{+}\right): 252.0540$ calcd for $\mathrm{C}_{11} \mathrm{H}_{11} \mathrm{~N}_{3} \mathrm{O}_{2} \mathrm{Cl} ; 252.0544$ found.

\subsubsection{Synthesis of methyl 2-(4-(3-chlorophenyl)-1H-1,2,3triazol-1-yl)acetate (3h)}

$\mathrm{R}_{f}=0.6\left(\right.$ Cyclohexane/EtOAc, 1:1, v/v); White solid; mp: 100-102 ${ }^{\circ} \mathrm{C}$; IR: $3127(\mathrm{CHar}), 1747(\mathrm{C}=\mathrm{O}$ ester); Yield = 68\%; ${ }^{1} \mathrm{H}$ NMR (300.13 MHz, DMSO) $8.73(\mathrm{~s}, 1 \mathrm{H}, \mathrm{C}=\mathrm{CH}-\mathrm{N}), 8.13(\mathrm{dd}, \mathrm{J}=7.6,1.9 \mathrm{~Hz}, 1 \mathrm{H}, \mathbf{H a r}), 7.59$ (dd, J = 7.8, $1.2 \mathrm{~Hz}, 1 \mathrm{H}$, Har), $7.52-7.36(\mathrm{~m}, 2 \mathrm{H}, \mathrm{Har}), 5.51\left(\mathrm{~s}, 2 \mathrm{H}, \mathrm{N}-\mathrm{CH}_{2}-\mathrm{C}=\mathrm{O}\right), 3.74\left(\mathrm{~s}, 3 \mathrm{H}, \mathrm{O}-\mathrm{CH}_{3}\right) ;{ }^{13} \mathrm{C}$ NMR $(75 \mathrm{MHz}, \mathrm{DMSO}) 167.6(\mathbf{C}=\mathrm{O})$, 145.0 (C-C-N=), 133.7 (Car), 132.5 (Car), 130.9 (CHar), 127.7 (CHar), 124.7 (CHar), 123.7 (CHar), 123.5 (C=CH-N), 52.6 $\left(\mathrm{O}-\mathrm{CH}_{3}\right), 50.5\left(\mathrm{~N}-\mathrm{CH}_{2}\right)$. HRMS $\left(\mathrm{M}+\mathrm{H}^{+}\right)$: 252.0540 calcd for $\mathrm{C}_{11} \mathrm{H}_{11} \mathrm{~N}_{3} \mathrm{O}_{2} \mathrm{Cl} ; 252.0543$ found.

\subsubsection{Synthesis of methyl 2-(4-(4-chlorophenyl)-1H-1,2,3triazol-1-yl)acetate (3i)}

$\mathrm{R}_{f}=0.4\left(\right.$ Cyclohexane/EtOAc, 1:1, v/v); White solid; mp: 143-145 ${ }^{\circ} \mathrm{C}$; IR: 3127 ( CHar), $1743(\mathrm{C}=\mathrm{O}$ ester); Yield = 27\%; ${ }^{1} \mathrm{H}$ NMR (300 MHz, DMSO) $8.62(\mathrm{~s}, 1 \mathrm{H}, \mathrm{C}=\mathrm{CH}-\mathrm{N}), 8.11-7.77$ (m, 2H, Har), $7.70-7.41$ (m, 2H, Har), 5.49 (s, 2H, N-CH $\mathbf{2}^{-}$ $\mathrm{C}=\mathrm{O}), 3.74$ (s, 3H, O-CH3); ${ }^{13} \mathrm{C}$ NMR (75 MHz, DMSO) $167.6(\mathbf{C}=\mathrm{O}), 145.3(\mathrm{C}-\mathbf{C}-\mathrm{N}=), 132.4(\mathbf{C a r}), 129.4$ (Car), 129.0 $(\mathbf{C H a r}), 126.8(\mathbf{C H a r}), 123.1(\mathrm{C}=\mathbf{C H}-\mathrm{N}), 52.6\left(\mathrm{O}-\mathrm{CH}_{3}\right), 50.5\left(\mathrm{~N}_{-} \mathrm{CH}_{2}\right)$. HRMS $\left(\mathrm{M}+\mathrm{H}^{+}\right): 252.0540$ calcd for $\mathrm{C}_{11} \mathrm{H}_{11} \mathrm{~N}_{3} \mathrm{O}_{2} \mathrm{Cl}$; 252.0542 found.

\subsubsection{Synthesis of methyl 2-(4-(2-fluorophenyl)-1H-1,2,3triazol-1-yl)acetate (3j)}

$\mathrm{R}_{f}=0.6\left(\right.$ Cyclohexane/EtOAc, 1:1, v/v); Pale brown solid; mp: 85-87 ${ }^{\circ} \mathrm{C}$; IR: $3169(\mathrm{CHar}), 1742(\mathrm{C}=\mathrm{O}$ ester); Yield = 10\%; ${ }^{1} \mathrm{H}$ NMR (300.13 MHz, DMSO) $8.49(\mathrm{~d}, \mathrm{~J}=3.9 \mathrm{~Hz}, 1 \mathrm{H}, \mathrm{C}=\mathrm{CH}-\mathrm{N}), 8.16(\mathrm{td}, \mathrm{J}=7.6,1.6 \mathrm{~Hz}, 1 \mathrm{H}, \mathbf{H a r})$,

$7.50-7.28$ (m, 3H, Har), 5.50 (s, 2H, N-CHz2-C=O), 3.73 (s, 3H, O-CH3); ${ }^{13} \mathrm{C}$ NMR (75 MHz, DMSO) $167.6(\mathbf{C}=\mathrm{O})$, 
158.4 (d, $J=247.0 \mathrm{~Hz}, \mathrm{~F}-\mathrm{Car}), 139.6$ (d, J = 2.0 Hz, C-CN=), 129.7 (d, J = 8.4 Hz, CHar), 127.2 (d, J = 3.2 Hz,

CHar), 125.2 (d, J = 11.7 Hz, C=CH-N), 125 (d, J = 2.9 Hz, CHar), 118.1 (d, J = 13.0 Hz, Car), 116.0 (d, J = 21.3 Hz, CHar), $52.5\left(\mathrm{O}-\mathrm{CH}_{3}\right), 50.4\left(\mathrm{~N}_{-} \mathrm{CH}_{2}\right) ;{ }^{19} \mathrm{~F}$ NMR $(282 \mathrm{MHz}, \mathrm{DMSO})$-114.8. HRMS $\left(\mathrm{M}+\mathrm{H}^{+}\right): 236.0835$ calcd for $\mathrm{C}_{11} \mathrm{H}_{11} \mathrm{~N}_{3} \mathrm{O}_{2} \mathrm{~F}$; 236.0837 found.

\subsubsection{Synthesis of methyl 2-(4-(3-fluorophenyl)-1H-1,2,3triazol-1-yl)acetate (3k)}

$\mathrm{R}_{f}=0.3$ (Cyclohexane/EtOAc, 1:1, v/v); White solid; mp: 113-115² ; IR: 3110 ( CHar), 1745 ( C=O ester); Yield = 18\%; ${ }^{1} \mathrm{H}$ NMR (300.13 MHz, DMSO) $8.64(\mathrm{~s}, 1 \mathrm{H}, \mathrm{C}=\mathrm{CHN}), 7.69(\mathrm{ddd}, \mathrm{J}=5.5,4.0,1.1 \mathrm{~Hz}, 2 \mathrm{H}, \mathrm{Har}), 7.51$ (td, J = 7.9, 6.2 Hz, 1H, Har), $7.25-7.07$ (m, 1H, Har), 5.49 (s, 2H, N-CH2 $-\mathrm{C}=\mathrm{O}), 3.74\left(\mathrm{~s}, 3 \mathrm{H}, \mathrm{O}-\mathrm{CH}_{3}\right)$; 13C NMR $(75 \mathrm{MHz}$, DMSO) $167.6(\mathbf{C}=\mathrm{O}), 162.6(\mathrm{~d}, \mathrm{~J}=243.2 \mathrm{~Hz}, \mathrm{~F}-\mathrm{Car}), 145.3(\mathrm{C}-\mathrm{C}-\mathrm{N}=), 132.8(\mathrm{~d}, \mathrm{~J}=8.5 \mathrm{~Hz}, \mathbf{C a r}), 131.1(\mathrm{~d}, \mathrm{~J}=8.5 \mathrm{~Hz}$, CHar), 123.4 (C=CH-N), 121.2 (d, J = 2.1 Hz, CHar), 114.7 (d, J = 21.1 Hz, CHar), 111.7 (d, J = 23.0 Hz, CHar), 52.6 (O$\left.\mathrm{CH}_{3}\right), 50.5(\mathrm{~N}-\mathrm{CH} 2) ;{ }^{19} \mathrm{~F}$ NMR (282 MHz, DMSO) -112.6. HRMS $\left(\mathrm{M}+\mathrm{H}^{+}\right): 236.0835$ calcd for $\mathrm{C}_{11} \mathrm{H}_{11} \mathrm{~N}_{3} \mathrm{O}_{2} \mathrm{~F} ; 236.0839$ found.

\subsubsection{Synthesis of methyl 2-(4-(4-fluorophenyl)-1H-1,2,3triazol-1-yl)acetate (3l)}

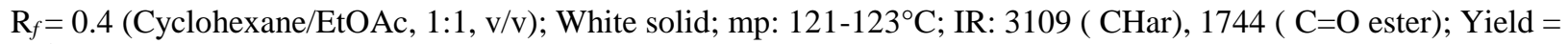
$66 \%$; ${ }^{1} \mathrm{H}$ NMR (300.13 MHz, DMSO) $8.56(\mathrm{~s}, 1 \mathrm{H}, \mathrm{C}=\mathrm{CHN}), 8.05-7.79(\mathrm{~m}, 2 \mathrm{H}, \mathbf{H a r}), 7.47-7.15(\mathrm{~m}, 2 \mathrm{H}, \mathbf{H a r}), 5.47(\mathrm{~s}, 2 \mathrm{H}$, $\mathrm{N}-\mathrm{CH} 2-\mathrm{C}=\mathrm{O}), 3.74$ (s, 3H, O-CH3); ${ }^{13} \mathrm{C}$ NMR

(75 MHz, DMSO) $167.6(\mathbf{C}=\mathrm{O}), 161.8(\mathrm{~d}, \mathrm{~J}=244.5 \mathrm{~Hz}, \mathrm{FCar}), 145.5(\mathrm{C}-\mathrm{C}-\mathrm{N}=), 127.2(\mathrm{~d}, \mathrm{~J}=8.2 \mathrm{~Hz}, \mathbf{C H a r}), 127(\mathrm{~d}, J=2.9$ $\mathrm{Hz}, \mathbf{C a r}), 122.6(\mathrm{C}=\mathrm{CH}-\mathrm{N}), 115.9(\mathrm{~d}, \mathrm{~J}=21.7 \mathrm{~Hz}, \mathbf{C H a r}), 52.6\left(\mathrm{O}-\mathrm{CH}_{3}\right), 50.4(\mathrm{~N}-\mathrm{CH} 2) ;{ }^{19} \mathrm{~F}$ NMR $(282 \mathrm{MHz}, \mathrm{DMSO})-113.9$. HRMS $\left(\mathrm{M}+\mathrm{H}^{+}\right): 236.0835$ calcd for $\mathrm{C}_{11} \mathrm{H}_{11} \mathrm{~N}_{3} \mathrm{O}_{2} \mathrm{~F} ; 236.0839$ found.

\subsubsection{Synthesis of methyl 2-(4-(3-hydroxyphenyl)-1H-}

\section{1,2,3-triazol-1-yl)acetate (3m)}

$\mathrm{R}_{f}=0.2$ (Cyclohexane/EtOAc, 1:1, v/v); White solid; mp: 131-133 C; IR: 3289 ( OH), 3051 ( CHar), 1729 ( C=O ester); Yield $=99 \% ;{ }^{1} \mathrm{H}$ NMR (300.13 MHz, DMSO) 9.57 (s, 1H, -OH), $8.49(\mathrm{~s}, 1 \mathrm{H}, \mathrm{C}=\mathrm{CH}-\mathrm{N}), 7.38-7.15$ (m,

$3 \mathrm{H}$, Har), $6.85-6.65$ (m, 1H, Har), 5.45 (s, 2H, N-CH2 $\mathrm{C}=\mathrm{O}), 3.73$ (s, 3H, O-CH3); ${ }^{13} \mathrm{C}$ NMR (75 MHz, DMSO) $168(\mathbf{C}=\mathrm{O})$, 157.7 (Car), 146.4 (C-C-N=), 131.6 (Car), 130 (CHar), 122.6 (C=CH-N), 116 (CHar), 115 (CHar), 111.8 (CHar), 52.5 (N$\left.\mathrm{CH}_{2}\right), 50.4\left(\mathrm{~N}-\mathrm{CH}_{2}\right)$. HRMS $\left(\mathrm{M}+\mathrm{H}^{+}\right): 234.0879$ calcd for $\mathrm{C}_{11} \mathrm{H}_{11} \mathrm{~N}_{3} \mathrm{O}_{3} ; 234.0880$ found.

\subsubsection{Synthesis of methyl methyl 2-(4-(thiophen-3-yl)-1H- \\ 1,2,3-triazol-1-yl)acetate(3n)}

$\mathrm{R}_{f}=0.4$ (Cyclohexane/EtOAc, 1:1, v/v); White solid; mp: 80-82 ${ }^{\circ} \mathrm{C}$; IR: 3111 ( CHar), 1731 ( $\mathrm{C}=\mathrm{O}$ ester); Yield $=80 \% ;{ }^{1} \mathrm{H}$ NMR (300.13 MHz, DMSO) $8.43(\mathrm{~s}, 1 \mathrm{H}, \mathrm{C}=\mathrm{CH}-\mathrm{N}), 7.89(\mathrm{~d}, \mathrm{~J}=2.9 \mathrm{~Hz}, 1 \mathrm{H}, \mathrm{Har}), 7.66(\mathrm{dd}, \mathrm{J}=5.0,2.9 \mathrm{~Hz}, 1 \mathrm{H}, \mathrm{Har}), 7.53$ $(\mathrm{d}, \mathrm{J}=5.0 \mathrm{~Hz}, 1 \mathrm{H}, \mathbf{H a r}), 5.47\left(\mathrm{~s}, 2 \mathrm{H}, \mathrm{N}-\mathrm{CH}_{2}-\mathrm{C}=\mathrm{O}\right), 3.73\left(\mathrm{~s}, 3 \mathrm{H}, \mathrm{O}-\mathrm{CH}_{3}\right) ;{ }^{13} \mathrm{C}$ NMR $(75 \mathrm{MHz}, \mathrm{DMSO}) 167.7(\mathbf{C}=\mathrm{O}), 142.9$ (C-C-N=), $131.8(\mathrm{C3}), 127.2$

(CHar), 125.7 (CHar), $122.4(\mathrm{CHar}), 120.9(\mathrm{CHar}), 52.5\left(\mathrm{NCH}_{2}\right), 50.3\left(\mathrm{~N}-\mathrm{CH}_{2}\right)$. HRMS $\left(\mathrm{M}+\mathrm{H}^{+}\right)$: 224.0494 calcd for $\mathrm{C}_{9} \mathrm{H}_{9} \mathrm{~N}_{3} \mathrm{O}_{2} \mathrm{~S} ; 224.0497$ found.

\subsubsection{Synthesis of methyl 2-(4-(pyridin-2-yl)-1H-1,2,3triazol-1-yl)acetate (3o)}

White solid; mp: 77-79 ${ }^{\circ} \mathrm{C}$; IR: 3135 ( CHar), 1752 ( $\mathrm{C}=\mathrm{O}$ ester); Yield $=100 \%$; ${ }^{1} \mathrm{H}$ NMR $(300.13 \mathrm{MHz}, \mathrm{DMSO}) 8.68-$ $8.46(\mathrm{~m}, 2 \mathrm{H}, \mathrm{C}=\mathrm{CH}-\mathrm{N}, \mathrm{Har}), 8.05(\mathrm{~d}, \mathrm{~J}=7.9 \mathrm{~Hz}, 1 \mathrm{H}, \mathrm{Har}), 7.90(\mathrm{td}, \mathrm{J}=7.7,1.8 \mathrm{~Hz}, 1 \mathrm{H}, \mathrm{Har}), 7.35$

(ddd, $\mathrm{J}=7.5,4.9,1.1 \mathrm{~Hz}, 1 \mathrm{H}, \mathrm{Har}), 5.49\left(\mathrm{~s}, 1 \mathrm{H}, \mathrm{N}-\mathrm{CH}_{2} \mathrm{C}=\mathrm{O}\right), 3.74\left(\mathrm{~s}, 2 \mathrm{H}, \mathrm{O}-\mathrm{CH}_{3}\right) ;{ }^{13} \mathrm{C}$ NMR $(75 \mathrm{MHz}, \mathrm{DMSO}) 167.6$ $(\mathrm{C}=\mathrm{O}), 149.7(\mathrm{C}-\mathrm{C}-\mathrm{N}=), 149.7$ (CHar), $147.2(\mathrm{Car}), 137.2(\mathrm{CHar}), 124.6(\mathrm{C}=\mathrm{CH}-\mathrm{N}), 123.0$ (CHar), 119.4 (CHar), 52.6 (O$\left.\mathrm{CH}_{3}\right), 50.4\left(\mathrm{~N}-\mathrm{CH}_{2}\right)$. HRMS $\left(\mathrm{M}+\mathrm{H}^{+}\right)$: 219.0882 calcd for $\mathrm{C}_{10} \mathrm{H}_{11} \mathrm{~N}_{4} \mathrm{O}_{2}$; found 219.0883 .

\subsubsection{Synthesis of methyl 2-(4-(pyridin-3-yl)-1H-1,2,3triazol-1-yl)acetate (3p)}

$\mathrm{R}_{f}=0.4$ (Cyclohexane/EtOAc, 1:1, v/v); White solid; $\mathrm{mp}: 104-106^{\circ} \mathrm{C}$; IR: $3110(\mathrm{CHar}), 1745\left(\mathrm{C}=\mathrm{O}\right.$ ester); Yield $=56 \% ;{ }^{1} \mathrm{H}$ NMR (300 MHz, DMSO) 9.07 (s, 1H, Har), $8.70(\mathrm{~s}, 1 \mathrm{H}, \mathrm{C}=\mathrm{CH}-\mathrm{N}), 8.56$ (d, J = 2.5 Hz, 1H, Har), 8.24 (dt, J = 7.9, 1.7 Hz, $1 \mathrm{H}$, Har), 7.50 (dd, J = 7.9, $4.8 \mathrm{~Hz}, 1 \mathrm{H}, \mathbf{H a r}), 5.52\left(\mathrm{~s}, 2 \mathrm{H}, \mathrm{N}-\mathrm{CH}_{2}-\mathrm{C}=\mathrm{O}\right.$ ), 3.74 (s, 3H, O-CH3); ${ }^{13} \mathrm{C}$ NMR (75 MHz, DMSO) 167.6 (C=O), $149(\mathbf{C H a r}), 146.3(\mathbf{C H a r}), 143.6(\mathrm{C}-\mathbf{C}-\mathrm{N}=)$, $132.4(\mathbf{C H a r}), 126.4(\mathbf{C a r}), 124.1(\mathbf{C H a r}), 123.4(\mathrm{C}=\mathbf{C H}-\mathrm{N}), 52.6$ $\left(\mathrm{O}-\mathrm{CH}_{3}\right), 50.5\left(\mathrm{~N}-\mathrm{CH}_{2}\right)$. HRMS $\left(\mathrm{M}+\mathrm{H}^{+}\right): 219.0882$ calcd for $\mathrm{C}_{10} \mathrm{H}_{11} \mathrm{~N}_{4} \mathrm{O}_{2}$; found 219.0884 .

\subsection{Computational Studies: Preparation of Enzyme and Compounds for Docking}

The crystal structure of Src kinase was extracted from Protein Data Bank (PDB code: 2SRC) [36]. All the water molecules and heteroatoms were removed and hydrogen atoms were added to the protein. The protein was completed and reprotonated 
with SPORE [37]. The chemical structures of all the synthesized compounds were generated using MarvinSketch and were subsequently converted into 3D format using Open Babel [38]. A series of docking experiment were carried out with all the designed triazoles derivatives against Src using PLANTS [39] for possible kinase inhibition activities. The compounds were selected on the basis of their scores and those reflecting good affinity were re-scored with DSX [40]. As a parameter for the molecular docking, the ant algorithm was set to 1 (highest reliability). The binding site was defined as a $18 \AA$ radius pocket centered on hinge's E339 in order to be sure that the inhibitors could fit in the kinase active site. The number of poses was set to 10 and the AMP-PNP was used as a shape constraint. The finally obtained docked complexes were subsequently visualized using PyMol.

\subsection{Biological Evaluations}

Src Kinase activity assays.

The effects of synthesized compounds on the activity of src kinase was determined by Z'Lyte biochemical assay kit from Life Technologies, according to manufacturer's protocol (Invitrogen, catalogue $n^{\circ} \mathrm{P} 3044$ ). Reactions were performed in 384 well low volume black non binding surface round bottom microplate (Corning $n^{\circ} 3676$ ). Briefly, test compounds were added to plate in the presence of ATP and specific substrates in Src kinase solution, and incubated for $1 \mathrm{~h}$ at room temperature before the addition of Development reagent solution. After the application of stabilizer solution, fluorescence polarization values were measured at 485/530 $\mathrm{nm}$ using a fluorescence reader. All compounds were dissolved in DMSO and diluted in buffer, yielding a final DMSO concentration of $1 \%$ in the assay.

\section{RESULTS AND DISCUSSION}

\subsection{Molecular Docking Analysis}

To achieve this goal within a reasonable timeframe, we needed a rapid and robust docking tool. Docking studies have been used previously to find novel inhibitors with different biological properties [41-43] in particular in the discovery of Src kinase inhibitors [44-47]. Our studies were performed with the software PLANTS as virtual screening software and 16 compounds were evaluated. The analysis of the docking results showed that two of the compounds, namely $\mathbf{3 b}$ and $\mathbf{3 m}$, have a good score (Fig. 1).

Fig. (1). Docking score of compounds (3a-3p) obtained from PLANTS.

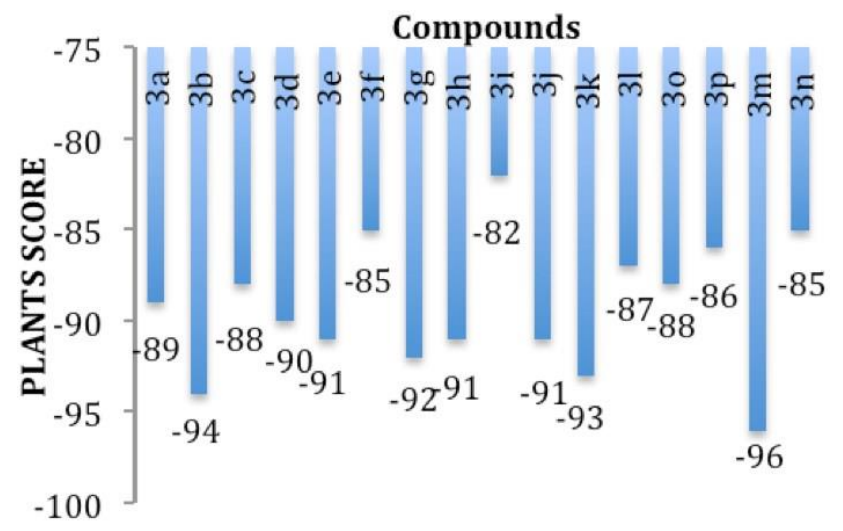

Both compounds show a hydrogen bond donor group in the meta position of the phenyl ring. All the compounds are in the ATP binding pocket and showed two different binding types on the hinge kinases. The first binding type is for compounds 3ac, 3m-p. The compounds 3a-c and $\mathbf{3 m}$ possess a hydrogen bond donor group and hence are capable of forming hydrogen bond with the hinge of Src kinase, especially with the C=O of GLU339 (Fig. 2a) and compounds 3n-p possess an acceptor group and show hydrogen bonding on the -NH group of MET341 (Fig. 2b). Compounds 3b and 3m also possess a good DSX prediction (Fig. 3).

The second binding mode involves the triazole moiety. Compounds 3d-i don't have any substituent able to establish hydrogen bonds, whereas compounds $\mathbf{3 j - 1}$ may be able to establish hydrogen bonds thanks to the fluorine group. In this case, one of the nitrogen of the triazole group form the hydrogen bond with the -NH group of MET341 (Fig. 4). 
a)

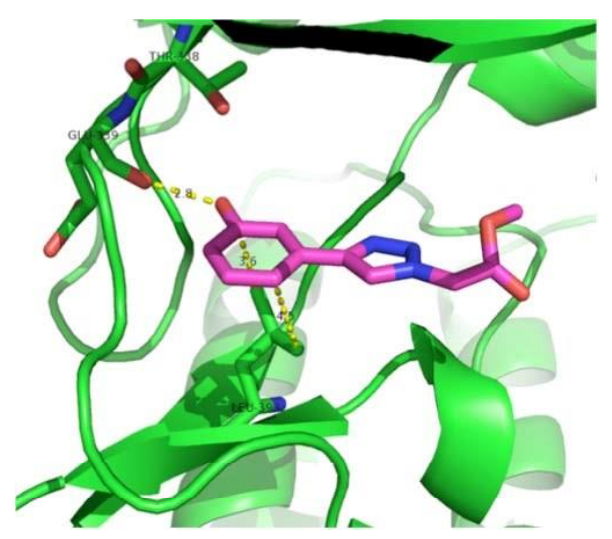

b)

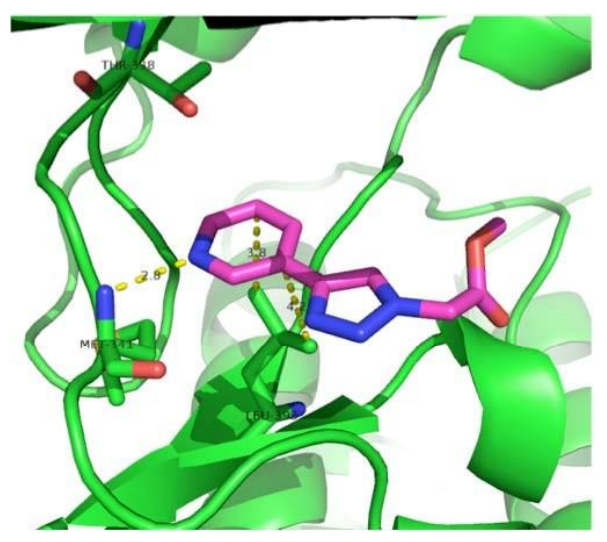

Fig. (2). Binding modes of compounds $3 \mathbf{m}$ and $\mathbf{3 p}$ calculated with PLANTS.

a) Binding mode of compound 3m calculated with PLANTS. The ligand is shown in pink and stick, c-Src (PDB 2SRC) is represented in green and cartoon, GLU339, gatekeeper residue THR338 and ILE393 are represented in sticks. The distance between the oxygen of the phenol group and the oxygen of the $-\mathrm{C}=\mathrm{O}$ of GLU339 equals to $2.8 \AA$ and hydrophobic interactions between ILE $\mathrm{CH}_{3}$ and pyridine moiety are between 3.6 and 4.5 $\mathrm{A}$. b) Binding mode of compound 3p calculated with PLANTS. The ligand is shown in pink and stick, c-Src (PDB 2SRC) is represented in green and cartoon, MET341 and ILE393 are represented in stick. The distance between the nitrogen of the pyridine group and the nitrogen of the $-\mathrm{NH}$ of MET341 equals to $2.8 \AA$ and hydrophobic interactions between $\mathrm{ILE} \mathrm{CH}_{3}$ and pyridine moiety are between 3.8 and $4.2 \AA$.

In our studies, we predicted that the synthesized molecules may be able to form hydrogen bond with the kinase hinge or with the gatekeeper residue, while earlier report showed modest inhibitory potency because of hydrophobic interactions [19].

\subsection{Chemistry}

For further development, we decided to synthesize the designed compounds by Huisgen's 1,3-dipolar cycloaddition (Scheme 1).

In method $\mathrm{A}$, the reaction of substituted terminal alkynes $\mathbf{1}$, methyl 2-azidoacetate 2, copper iodide (5 mol \%) and $\mathrm{NaAscorbate}$, in a mixture of solvent t- $\mathrm{BuOH} / \mathrm{H}_{2} \mathrm{O}$, at room temperature, afforded the 1,4-disubstituted 1,2,3-triazoles 3a-3p in moderate 3c-3l, 3p to good yield $\mathbf{3 a - b}, \mathbf{3 m - \mathbf { o }}$ after purification by column chromatography (Scheme 1, Table 2). 
a)

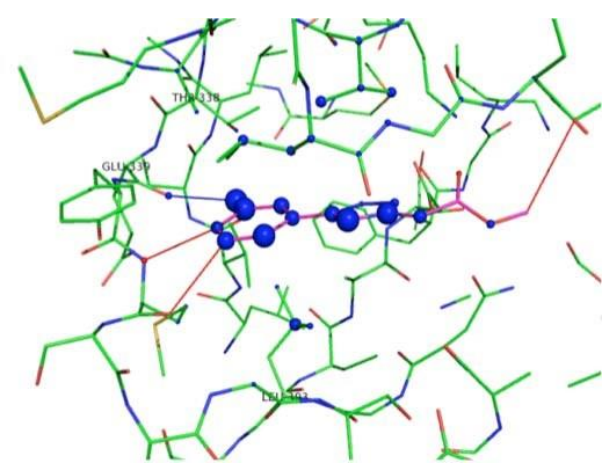

b)

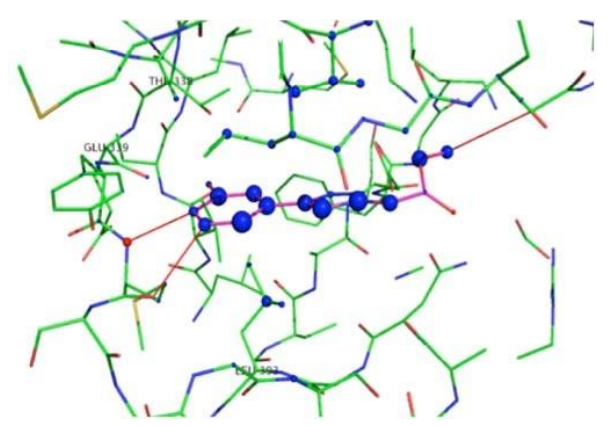

Fig. (3). DSX prediction for compounds 3b (a) and 3m (b). DSX prediction for compounds $\mathbf{3 b}$ and $\mathbf{3 m}$ blue spheres represents favorable interactions, red spheres represent bad interactions, blue lines represent good distances and red lines bad distances.

(a)

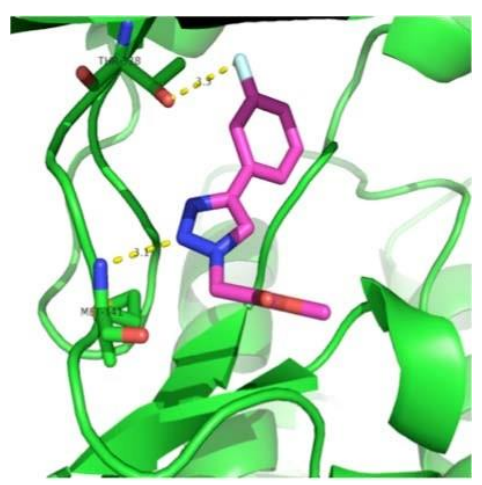

(b)

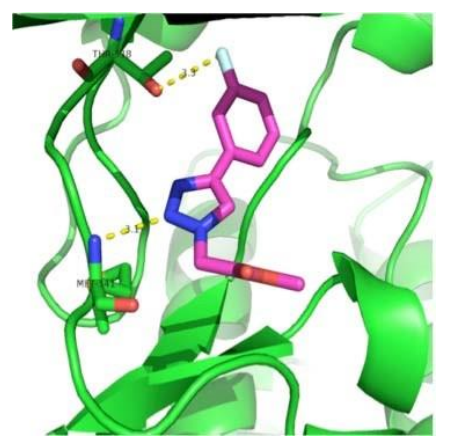

Fig. (4). Binding mode of compound 3k calculated with PLANTS (a) and DSX evaluation (b).

(a) Binding mode of compound 3k calculated with PLANTS. The ligand is shown in pink and stick, c-Src (PDB 2SRC) is represented in green and cartoon, MET341 and the gatekeeper residue THR338 are represented in sticks. The distance between the nitrogen of the triazole group and the nitrogen of the $-\mathrm{NH}$ of MET341 equals to 3.1 $\mathrm{A}$. (b) DSX prediction for compound $\mathbf{3 k}$. 


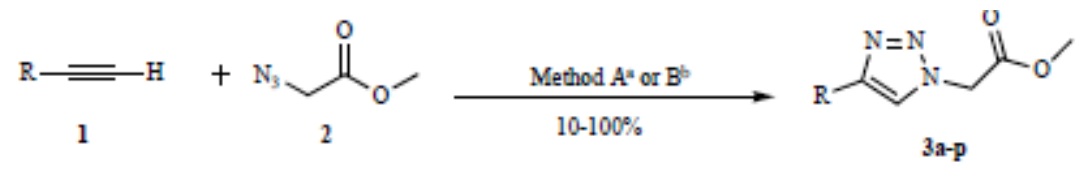

Method A : CuI (5 mol \%), t-BuOH/H$/ 2$ (1:1, V/V), rt 12-72 h

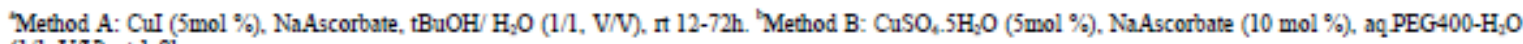
$(1 / 1, \mathrm{~V} / \mathrm{V})$, rt l-sh.

Scheme 1. Synthesis of Substituted 1,4-disubstituted 1,2,3-triazoles 3a-p.

Table 1. Chemical structures, chemical data and purity of synthesized compounds 3 .

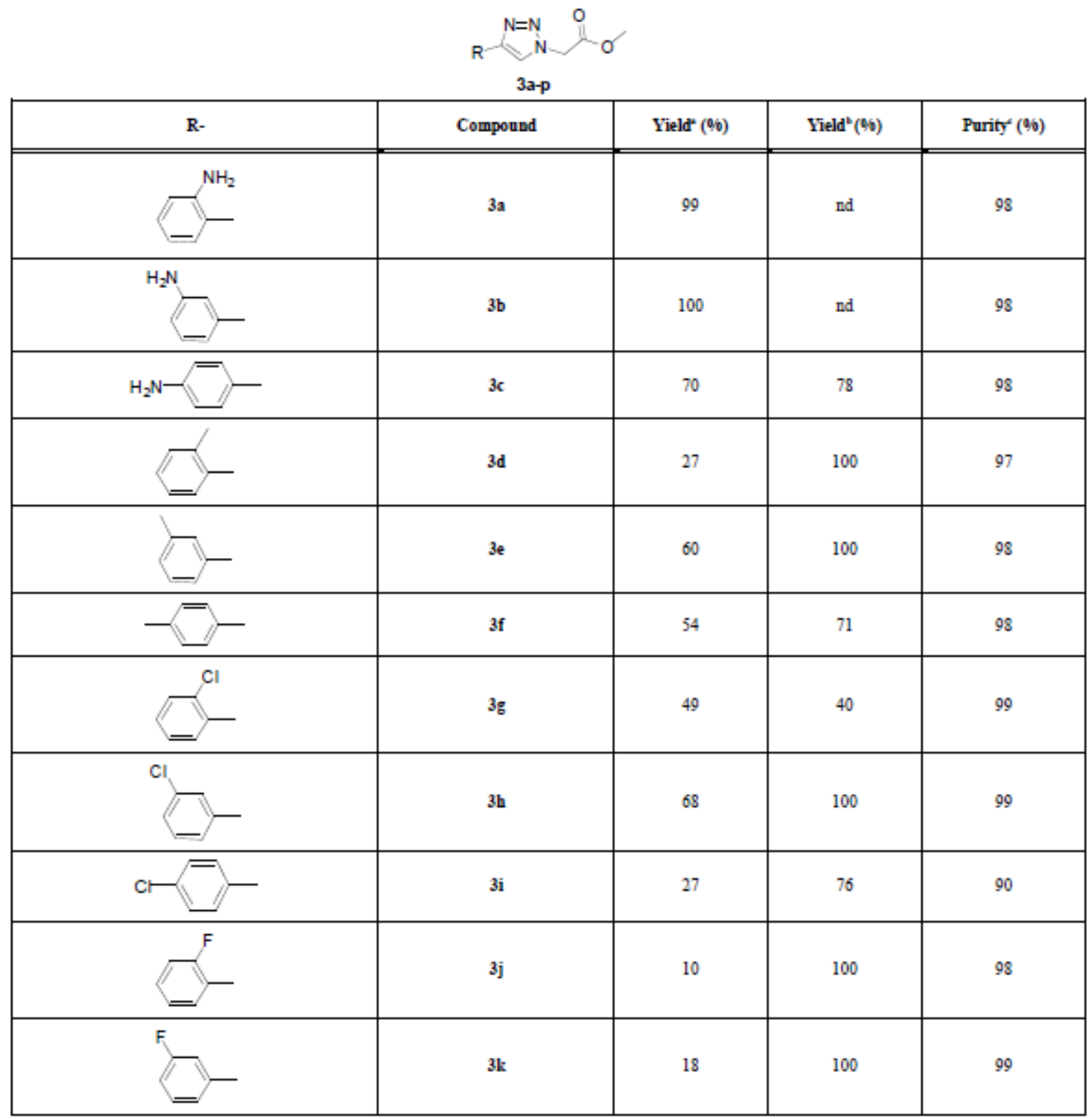


Table 1. contd...

\begin{tabular}{|c|c|c|c|c|}
\hline R- & Compound & $\begin{array}{c}\text { Yield }^{\mathrm{a}} \\
(\%)\end{array}$ & Yield $^{\mathrm{b}}(\%)$ & $\operatorname{Purity}^{\mathrm{c}}(\%)$ \\
\hline $\mathrm{F}$ & 31 & 66 & 100 & 99 \\
\hline $\mathrm{HO}$ & $3 m$ & 99 & nd & 98 \\
\hline & $3 n$ & 80 & nd & 99 \\
\hline & 30 & 100 & nd & 96 \\
\hline & $3 p$ & 56 & 30 & 99 \\
\hline
\end{tabular}

${ }^{\text {a } Y i e l d s ~ o b t a i n e d ~ b y ~ m e t h o d ~ A ~ a f t e r ~ p u r i f i c a t i o n ~ o f ~ c o m p o u n d s ~ b y ~ c o l u m n ~ f l a s h ~ c h r o m a t o g r a p h y ~}$

${ }^{b}$ Yields obtained by method B after purification of compounds by recrystallisation ${ }^{\mathrm{c}}$ HPLC Purity

compound containing hydroxyl group in the meta position on the phenyl ring $\mathbf{3 m}$ exhibited a more potent inhibitory activity $(27 \%)$ than the rest of the compounds suggesting that prediction from the molecular docking analysis is right. On the opposite, compound 3p showed weak inhibitory activity on Src kinase. This activity does not correlate with the docking results, since no activity was predicted. This can be explained by the limitations of the docking calculating method, which is fast and rough. More accurate results can be obtained by MM-PBSA calculations. However, this method takes much more time and computing capacity. Unfortunately, none of the synthesized compounds exhibited an activity similar to dasatinib.

Table 2. The Src kinase inhibitory activity of compounds 3a-p.

The synthesized triazoles $\mathbf{3}$ are completely characterized by ${ }^{1} \mathrm{H},{ }^{13} \mathrm{C},{ }^{19} \mathrm{~F}$ NMR, IR, HPLC and HRMS. The formation of triazole was confirmed by the presence of an absorption band in the region of $3051-3169 \mathrm{~cm}^{-1}$ in IR spectra due to the $=\mathrm{CH}$ streching of triazole ring. The presence of characteristic singlet in ${ }^{1} \mathrm{H}$ NMR due to triazolyl protons in the region of 8.25-8.73 ppm and 122.28-125.69 ppm in ${ }^{13} \mathrm{C}$ NMR due to $\mathrm{C}-5$ of the triazole ring confirmed the formation of the triazole ring.

Except 3f [49] and 3o [50], all the other 1,2,3-triazoles have not been described before.

\subsection{Biological Evaluations}

The 16 diversely substituted 1,2,3-triazoles were evaluated against Src kinase (Table 2). Dasatinib (commercially available dual SRc/Abl kinase inhibitor) was used as a positive control in the assays. The results of Src kinase inhibitory activity of compounds are shown in Table 3. The compounds with alkyl 3d-f, halogen 3g-l, amines 3a-c substituent on phenyl ring, thiophene $\mathbf{3 n}$ and two pyridines 3o-p exhibited only weak Src kinase inhibition with \% inhibition value less than $10 \%$. On the opposite, the

\begin{tabular}{|c|c|}
\hline Compound & \% Inhibition at 75M \pm sd \\
\hline \hline 3a & $5 \pm 2.76$ \\
\hline $3 \mathbf{b}$ & $4 \pm 0.53$ \\
\hline $\mathbf{3 c}$ & $4 \pm 0.57$ \\
\hline $\mathbf{3 d}$ & $7 \pm 1.13$ \\
\hline $\mathbf{3 e}$ & $-1 \pm 0.76$ \\
\hline $\mathbf{3 f}$ & $3 \pm 1.50$ \\
\hline $\mathbf{3 g}$ & $0 \pm 1.39$ \\
\hline $\mathbf{3 h}$ & $0 \pm 5.14$ \\
\hline $\mathbf{3 i}$ & $-5 \pm 3.63$ \\
\hline
\end{tabular}




\begin{tabular}{|c|c|}
\hline $\mathbf{3 j}$ & $7 \pm 5.84$ \\
\hline $\mathbf{3 k}$ & $1 \pm 0.61$ \\
\hline $\mathbf{3 m}$ & $-5 \pm 1.57$ \\
\hline $\mathbf{3 n}$ & $27 \pm 1.38$ \\
\hline $\mathbf{3 o}$ & $7 \pm 0.41$ \\
\hline $\mathbf{3 p}$ & $1 \pm 3.81$ \\
\hline Dasatinib & $11 \pm 3.39$ \\
\hline
\end{tabular}

\section{CONCLUSION}

A series of 1,4-disubstituted 1,2,3-triazole compounds were synthesized through an easy, convenient $\mathrm{Cu}(\mathrm{I})$ catalyzed click reaction and evaluated for their Src kinase activity. Compound 3m exhibited significant inhibitory activity against Src Kinase. These results, along with molecular design docking observations, are significant evidence to demonstrate the compound $\mathbf{3 m}$ could be optimized as a potential Src kinase inhibitor in further studies.

\section{CONFLICT OF INTEREST}

The authors declare that they have no conflict of interest. We gratefully thank the French "ministère de l'éducation nationale, de l'enseignement supérieur et de la recherche" and the University of Nîmes for their financial support.

\section{ACKNOWLEDGEMENTS}

Declared none.

\section{SUPPLEMENTARY MATERIAL}

Supplementary material is available on the publishers Web site along with the published article.

\section{REFERENCES}

[1] Miller, M. J.; Foy, K. C.; Kaumaya, P. T. P. Cancer Immunotherapy: Present Status, Future Perspective, and a New Paradigm of Peptide Immunotherapeutics. Discov. Med., 2013, 15, 166-176.

[2] Biscardi, J. S.; Tice, D. A.; Parsons, S. J. C-Src, Receptor Tyrosine Kinases, and Human Cancer. Adv. Cancer Res., 1999, 76, 61-119.

[3] Irby, R. B.; Yeatman, T. J. Role of Src Expression and Activation in Human Cancer. Oncogene., 2000, 19, 5636-5642.

[4] Schlessinger, J. Cell Signaling by Receptor Tyrosine Kinases. Cell, 2000, 103, 211-225.

[5] Martin, G. S. The Hunting of the Src. Nat. Rev. Mol. Cell Biol., 2001, 2, 467-475.

[6] Barrios Sosa, A. C.; Boschelli, D. H.; Wu, B.; Wang, Y.; Golas, J. M. Further Studies on Ethenyl and Ethynyl-4-Phenylamino3Quinolinecarbonitriles: Identification of a Subnanomolar Src Kinase Inhibitor. Bioorg. Med. Chem. Lett., 2005, 15, 1743-1747.

[7] Boschelli, D. H.; Wu, B.; Ye, F.; Wang, Y.; Golas, J. M.; Lucas, J.; Boschelli, F. Synthesis and Src Kinase Inhibitory Activity of a Series of 4-[(2,4-Dichloro-5-Methoxyphenyl)amino]-
7-Furyl-3Quinolinecarbonitriles. J. Med. Chem., 2006, 49, 78687876.

[8] Boschelli, D. H.; Wu, B.; Ye, F.; Durutlic, H.; Golas, J. M.; Lucas, J.; Boschelli, F. Facile Preparation of New 4-Phenylamino3Quinolinecarbonitrile Src Kinase Inhibitors via 7-Fluoro Intermediates: Identification of Potent 7-Amino Analogs. Bioorg. Med. Chem., 2008, 16, 405-412.

[9] Boschelli, D. H.; Wang, D.; Wang, Y.; Wu, B.; Honores, E. E.; Barrios Sosa, A. C.; Chaudhary, I.; Golas, J.; Lucas, J.; Boschelli, F. Optimization of 7-Alkene-3-Quinolinecarbonitriles as Src Kinase Inhibitors. Bioorg. Med. Chem. Lett., 2010, 20, 2924-2927.

[10] Nam, N.-H.; Lee, S.; Ye, G.; Sun, G.; Parang, K. ATP-

Phosphopeptide Conjugates as Inhibitors of Src Tyrosine Kinases. Bioorg. Med. Chem., 2004, 12, 5753-5766.

[11] Sundaramoorthi, R.; Shakespeare, W. C.; Keenan, T. P.; Metcalf, C. A.; Wang, Y.; Mani, U.; Taylor, M.; Liu, S.; Bohacek, R. S.; Narula, S. S.; Dalgarno, D. C.; van Schravandijk, M. R.; Violette, S. M.; Liou, S.; Adams, S.; Ram, M. K.; Keats, J. A.; Weigle, M.; Sawyer, T. K.; Weigele, M. Bone-Targeted Src Kinase Inhibitors: Novel Pyrrolo- and Pyrazolopyrimidine Analogues. Bioorg. Med. Chem., Lett., 2003, 13, 3063-3066.

[12] Wang, Y.; Metcalf, C. A.; Shakespeare, W. C.; Sundaramoorthi, R.; Keenan, T. P.; Bohacek, R. S.; van Schravendijk, M. R.; Violette, S. M.; Narula, S. S.; Dalgarno, D. C.; Haraldson, C.; Keats, J.; Liou, S.; Mani, U.; Pradeepan, S.; Ram, M.; Adams, S.; Weigele, M.; Sawyer, T. K. Bone-Targeted 2,6,9-Trisubstituted Purines: Novel Inhibitors of Src Tyrosine Kinase for the Treatment of Bone Diseases. Bioorg. Med. Chem. Lett., 2003, 13, 3067-3070.

[13] Huang, W.-S.; Zhu, X.; Wang, Y.; Azam, M.; Wen, D.; Sundaramoorthi, R.; Thomas, R. M.; Liu, S.; Banda, G.; Lentini, S. P.; Das, S.; Xu, Q.; Keats, J.; Wang, F.; Wardwell, S.; Ning, Y.; Snodgrass, J. T.; Broudy, M. I.; Russian, K.; Daley, G. Q.; Iuliucci, J.; Dalgarno, D. C.; Clackson, T.; Sawyer, T. K.; Shakespeare, W. C. 9-(Arenethenyl)purines as Dual Src/Abl Kinase Inhibitors Targeting the Inactive Conformation: Design, Synthesis, and Biological Evaluation. J. Med. Chem., 2009, 52, 4743-4756.

[14] Mukaiyama, H.; Nishimura, T.; Kobayashi, S.; Ozawa, T.; Kamada, N.; Komatsu, Y.; Kikuchi, S.; Oonota, H.; Kusama, H. Synthesis and c-Src Inhibitory Activity of imidazo[1,5-A]pyrazine Derivatives as an Agent for Treatment of Acute Ischemic Stroke. Bioorg. Med. Chem., 2007, 15, 868-885.

[15] Noronha, G.; Barrett, K.; Boccia, A.; Brodhag, T.; Cao, J.; Chow, C. P.; Dneprovskaia, E.; Doukas, J.; Fine, R.; Gong, X.; Gritzen, C.; Gu, H.; Hanna, E.; Hood, J. D.; Hu, S.; Kang, X.; Key, J.; Klebansky, B.; Kousba, A.; Li, G.; Lohse, D.; Mak, C. C.; McPherson, A.; Palanki, M. S. S.; Pathak, V. P.; Renick, J.; Shi, F.; Soll, R.; Splittgerber, U.; Stoughton, S.; Tang, S.; Yee, S.; Zeng, B.; Zhao, N.; Zhu, H. Discovery of [7-(2,6-Dichlorophenyl)5Methylbenzo [1,2,4]triazin-3-Yl]-[4-(2-Pyrrolidin1Ylethoxy)phenyl]amine--a Potent, Orally Active Src Kinase Inhibitor with Anti-Tumor Activity in Preclinical Assays. Bioorg. Med. Chem. Lett., 2007, 17, 602-608.

[16] Boschelli, D. H.; Powell, D.; Golas, J. M.; Boschelli, F. Inhibition of Src Kinase Activity by 4-Anilino-5,10-Dihydropyrimido[4,5B]quinolines. Bioorg. Med. Chem. Lett., 2003, 13, 2977-2980.

[17] Vu, C. B.; Luke, G. P.; Kawahata, N.; Shakespeare, W. C.; Wang, Y.; Sundaramoorthi, R.; Metcalf, C. A.; Keenan, T. P.; Pradeepan, S.; Corpuz, E.; Merry, T.; Bohacek, R. S.; Dalgarno, D. C.; Narula, S. S.; van Schravendijk, M. R.; Ram, M. K.; Adams, S.; Liou, S.; Keats, J. A.; Violette, S. M.; Guan, W.; Weigele, M.; Sawyer, T. K. Bone-Targeted pyrido[2,3-D]pyrimidin-7-Ones: Potent Inhibitors of Src Tyrosine Kinase as Novel Antiresorptive Agents. Bioorg. Med. Chem. Lett., 2003, 13, 3071-3074.

[18] Barlaam, B.; Fennell, M.; Germain, H.; Green, T.; Hennequin, L.; Morgentin, R.; Olivier, A.; Plé, P.; Vautier, M.; Costello, G. New Heterocyclic Analogues of 4-(2-Chloro-5Methoxyanilino)quinazolines as Potent and Selective c-Src Kinase Inhibitors. Bioorg. Med. Chem. Lett., 2005, 15, 5446-5449.

[19] Kumar, D.; Reddy, V. B.; Kumar, A.; Mandal, D.; Tiwari, R.; Parang, K. Click Chemistry Inspired One-Pot Synthesis of 
1,4Disubstituted 1,2,3-Triazoles and Their Src Kinase Inhibitory Activity. Bioorg. Med. Chem. Lett., 2011, 21, 449-452.

[20] Demaray, J. A.; Thuener, J. E.; Dawson, M. N.; Sucheck, S. J. Synthesis of Triazole-Oxazolidinones via a One-Pot Reaction and Evaluation of Their Antimicrobial Activity. Bioorg. Med. Chem. Lett., 2008, 18, 4868-4871.

[21] Gaur, M.; Goel, M.; Sridhar, L.; Ashok, T. D. S.; Prabhakar, S.; Dureja, P.; Raghunathan, P.; Eswaran, S. V. Synthesis, Characterization, and Antifungal Activity of Biaryl-Based bis(1,2,3Triazoles) Using Click Chemistry. Monatshefte Für Chem. - Chem. Mon., 2011, 143, 283-288.

[22] Lal, K.; Kumar, A.; Pavan, M. S.; Kaushik, C. P. Regioselective Synthesis and Antimicrobial Studies of Ester Linked 1,4Disubstituted 1,2,3-Bistriazoles. Bioorg. Med. Chem. Lett., 2012, 22, 4353-4357.

[23] D'hooghe, M.; Vandekerckhove, S.; Mollet, K.; Vervisch, K.; Dekeukeleire, S.; Lehoucq, L.; Lategan, C.; Smith, P. J.; Chibale, K.; De Kimpe, N. Synthesis of 2-Amino-3-Arylpropan-1-Ols and 1(2,3-Diaminopropyl)-1,2,3-Triazoles and Evaluation of Their Antimalarial Activity. Beilstein J. Org. Chem., 2011, 7, 1745-1752.

[24] Buckle, D. R.; Rockell, C. J.; Smith, H.; Spicer, B. A. Studies on 1,2,3-Triazoles. 13. (Piperazinylalkoxy) [1]benzopyrano[2,3D]1,2,3-Triazol-9(1H)-Ones with Combined H1-Antihistamine and Mast Cell Stabilizing Properties. J. Med. Chem., 1986, 29, 2262267.

[25] Vicentini, C. B.; Brandolini, V.; Guarneri, M.; Giori, P. Pyrazolo[3,4-d][1,2,3]triazole-1-Carboxamides and 5alkylaminopyrazolo[3,4-D]oxazoles: Synthesis and Evaluation of the in Vitro Antifungal Activity. Farm. Soc. Chim. Ital., 1992, 47, 1021-1034.

[26] Fung-Tomc, J. C.; Huczko, E.; Minassian, B.; Bonner, D. P. In Vitro Activity of a New Oral Triazole, BMS-207147 (ER-30346). Antimicrob. Agents Chemother., 1998, 42, 313-318.

[27] Whiting, M.; Muldoon, J.; Lin, Y.-C.; Silverman, S. M.; Lindstrom, W.; Olson, A. J.; Kolb, H. C.; Finn, M. G.; Sharpless, K. B.; Elder, J. H.; Fokin, V. V. Inhibitors of HIV-1 Protease by Using in Situ Click Chemistry. Angew. Chem. Int. Ed Engl., 2006, 45, 14351439.

[28] Cichero, E.; Buffa, L., Fossa, P. 3,4,5-Trisubstituted-1,2,44Htriazoles as WT and Y188L mutant HIV-1 non-nucleoside reverse transcriptase inhibitors: docking-based CoMFA and CoMSIA analyses, J. Mol. Model., 2011, 17, 1537-1550.

[29] Labadie, G. R.; de la Iglesia, A.; Morbidoni, H. R. Targeting Tuberculosis through a Small Focused Library of 1,2,3-Triazoles. Mol. Divers., 2011, 15, 1017-1024.

[30] Salmon, A. J.; Williams, M. L.; Wu, Q. K.; Morizzi, J.; Gregg, D.; Charman, S. A.; Vullo, D.; Supuran, C. T.; Poulsen, S.-A. Metallocene-Based Inhibitors of Cancer-Associated Carbonic Anhydrase Enzymes IX and XII. J. Med. Chem., 2012, 55, 55065517.

[31] Chinthala, Y.; Thakur, S.; Tirunagari, S.; Chinde, S.; Domatti, A. K.; Arigari, N. K.; K V N S, S.; Alam, S.; Jonnala, K. K.; Khan, F.; Tiwari, A.; Grover, P. Synthesis, Docking and ADMET Studies of Novel Chalcone Triazoles for Anti-Cancer and Anti-Diabetic Activity. Eur. J. Med. Chem., 2015, 93, 564-573.

[32] Bourne, Y.; Kolb, H. C.; Radi, Z.; Sharpless, K. B.; Taylor, P.; Marchot, P. Freeze-Frame Inhibitor Captures Acetylcholinesterase in a Unique Conformation. Proc. Natl. Acad. Sci. U. S. A., 2004 $101,1449-1454$

[33] Huisgen, R. 1,3-Dipolar Cycloadditions. Past and Future, Angew. Chem. Int. Ed. Engl., 1963, 2, 565-598.

[34] Tornøe, C. W.; Christensen, C.; Meldal, M. Peptidotriazoles on Solid Phase: [1,2,3]-Triazoles by Regiospecific Copper(i)Catalyzed
1,3-Dipolar Cycloadditions of Terminal Alkynes to Azides. J. Org Chem., 2002, 67, 3057-3064.

[35] Rostovtsev, V. V.; Green, L. G.; Fokin, V. V.; Sharpless, K. B. A Stepwise Huisgen Cycloaddition Process: copper(I)-Catalyzed Regioselective "Ligation" of Azides and Terminal Alkynes. Angew. Chem. Int. Ed Engl., 2002, 41, 2596-2599.

[36] Xu, W.; Doshi, A.; Lei, M.; Eck, M. J.; Harrison, S. C. Crystal Structures of c-Src Reveal Features of Its Autoinhibitory Mechanism. Mol. Cell, 1999, 3, 629-638.

[37] Brink, T. ten; Exner, T. E. Influence of Protonation, Tautomeric, and Stereoisomeric States on Protein-Ligand Docking Results. $J$. Chem. Inf. Model., 2009, 49, 1535-1546.

[38] O’Boyle, N. M.; Banck, M.; James, C. A.; Morley, C.; Vandermeersch, T.; Hutchison, G. R. Open Babel: An Open Chemical Toolbox. J. Cheminformatics., 2011, 3, 33.

[39] Korb, O.; Stützle, T.; Exner, T. E. Empirical Scoring Functions for Advanced Protein-Ligand Docking with PLANTS. J. Chem. Inf. Model., 2009, 49, 84-96.

[40] G. Neudert, G. Klebe, DSX: a knowledge-based scoring function for the assessment of protein-ligand complexes. J. Chem. Inf. Model., 2011, 51, 2731-2745.

[41] Baig, M.H. ; Ahmad, K.; Roy,S.; Ashraf, J.M.; Adil, M.; Siddiqui, M.H. Khan, S.: Kamal MA.; Provaznik, I., Choi, I. Computer Aided Drug Design: Success and Limitations. Curr. Pharm. Des., 2016, 22, 572-581.

[42] Danishuddin,M. ; Khan, A.; Faheem, M.; Kalaiarasan, P.; Hassan Baig, M.; Subbarao, N.; Khan, AU. Structure-based screening of inhibitors against KPC-2: designing potential drug candidates against multidrug-resistant bacteria. J. Biomol. Struct. Dyn., 2014, 32, 741-750.

[43] Khan, M.S.; Baig, M.H.; Ahmad, S.; Siddiqui, S.A. ; Srivastava, A.K. ; Srinivasan, K.V. ; Ansari, IA. Design, synthesis, evaluation and thermodynamics of 1-substituted pyridylimidazo[1,5a]pyridine derivatives as cysteine protease inhibitors. PloS One. 2013, 8 , e69982.

[44] Cui, Z. ; Li, X. ; L. Li, Zhang, B. ; Gao, C. ; Chen, Y.; Tan, C.; Liu, H.; Xie, W.; Yang, T.; Jiang, Y. Design, synthesis and evaluation of acridine derivatives as multi-target Src and MEK kinase inhibitors for anti-tumor treatment. Bioorg. Med. Chem., 2016, 24, 261269.

[45] Francini, C.M.; Fallacara, A.L.; Artusi, R. ; Mennuni, L.; Calgani, A. Angelucci, A.; Schenone, S.; Botta, M. Identification of Aminoimidazole and Aminothiazole Derivatives as Src Family Kinase Inhibitors. ChemMedChem, 2015, 10, 2027-2041.

[46] Z. Kilic-Kurt, F. Bakar, S. Ölgen, Synthesis, Biological, and Computational Evaluation of Novel 1,3,5-Substituted Indolin-2-one Derivatives as Inhibitors of Src Tyrosine Kinase. Arch. Pharm. (Weinheim)., 2015, 348, 2015, 715-729.

[47] B. Wright, K.A. Watson, L.J. McGuffin, J.A. Lovegrove, J.M. Gibbins, GRID and docking analyses reveal a molecular basis for flavonoid inhibition of Src family kinase activity. J. Nutr. Biochem. 2015, 26, 1156-1165.

[48] Kumar, D.; Reddy, V. B.; Varma, R. S. A Facile and Regioselective Synthesis of 1,4-Disubstituted 1,2,3-Triazoles Using Click Chemistry. Tetrahedron Lett., 2009, 50, 2065-2068

[49] Buckley, B. R.; Dann, S. E.; Heaney, H.; Stubbs, E. C. Heterogeneous Catalytic Reactions "On Water" by Using Stable Polymeric Alkynylcopper(I) Pre-Catalysts: Alkyne/Azide Cycloaddition Reactions. Eur. J. Org. Chem., 2011, 2011, 770-776.

[50] François, C. Auzanneau, V. Le Morvan, C. Galaup, H.S. Godfrey, L. Marty, et al., A functionalized heterobimetallic $(99 \mathrm{~m}) \mathrm{Tc} / \mathrm{Re}$ complex as a potential dual-modality imaging probe: synthesis,

photophysical properties, cytotoxicity and cellular imaging investigations. Dalton Trans., 2014, 43, 439-450. 